Meta

Journal des traducteurs

Translators' Journal

\title{
Traduction et stylistique : Une analyse de la traduction d'Arrow of God de Chinua Achebe
}

\section{Iheanacho A. Akakuru et Nwanne Mkpa}

Volume 42, numéro 4, décembre 1997

URI : https://id.erudit.org/iderudit/001865ar

DOI : https://doi.org/10.7202/001865ar

Aller au sommaire du numéro

Éditeur(s)

Les Presses de l'Université de Montréal

ISSN

0026-0452 (imprimé)

1492-1421 (numérique)

Découvrir la revue

Citer cet article

Akakuru, I. A. \& Mkpa, N. (1997). Traduction et stylistique : Une analyse de la traduction d'Arrow of God de Chinua Achebe. Meta, 42(4), 641-648.

https://doi.org/10.7202/001865ar

\section{Résumé de l'article}

Les auteurs analysent la traduction a Arrow of God afin de déterminer dans quelle mesure le processus de traduction a influencé le style, voire l'orientation de l'œuvre originale. Us relèvent des cas de modifications de la langue source qui concernent le plus souvent des mots I expressions qui sont

contexte-dépendants et concluent que même si, dans l'ensemble, la traduction respecte l'esthétique de l'original, on remarque néanmoins un décentrement. Or, les éléments que l'on violente sont ceux qui participent du style de l'auteur, de ses stratégies particulières, etc. Et c'est en les modifiant, en cherchant à leur imposer une nouvelle individualité, que le traducteur porte atteinte à l'intégrité de l'original. 


\title{
TRADUCTION ET STYLISTIQUE : UNE ANALYSE DE LA TRADUCTION D'ARROW OF GOD DE CHINUA ACHEBE
}

\author{
IHEANACHO A. AKAKURU ET NWANNE MKPA \\ Université de Port Harcourt, Port Harcourt et \\ Akanu Ibiam Polytechnic, Uwana-Afikpo, Nigeria
}

\begin{abstract}
Résumé
Les auteurs analysent la traduction d'Arrow of God afin de déterminer dans quelle mesure le processus de traduction a influencé le style, voire l'orientation de l'auvre originale. Ils relèvent des cas de modifications de la langue source qui concernent le plus souvent des mots / expressions qui sont contexte-dépendants et concluent que même si, dans l'ensemble, la traduction respecte l'esthétique de l'original, on remarque néanmoins un décentrement. Or, les éléments que l'on violente sont ceux qui participent du style de l'auteur, de ses stratégies particulières, etc. Et c'est en les modifiant, en cherchant à leur imposer une nouvelle individualité, que le traducteur porte atteinte à l'intégrité de l'original.
\end{abstract}

\begin{abstract}
An analysis of the translation of the "Arrow of God" is used to show the extent to which the translation process has influenced the style and general orientation of the original work. The authors cite examples of changes to the source text relating primarily to context dependent words and expressions and conclude that even though in a general sense the translation reflects the esthetics of the original, it has been "decentred". By tampering with the author's style, and giving it a new personality, the translator has violated the integrity of the original.
\end{abstract}

\section{INTRODUCTION}

La traduction des textes est devenue, de nos jours, un impératif existentiel. Le besoin de communication interlinguale, la nécessité pour l'humanité — pour son bien-être — de se rapprocher du «village global» de McLuhan', imposent aux sociétés contemporaines d'amener à la lisibilité (voire à l'intelligibilité des autres) des textes autrement codés en langues étrangères. En effet, la traduction pose tour à tour les problèmes de la coïncidence/non-coincidence totale de deux langues, du message/transfert du message ${ }^{2}$. Car, si nous posons pour le texte scientifique une univocité du signe linguistique, on ne peut ne pas prendre en compte, pour la littérature, l'instabilité proverbiale de l'énoncé. Et cela, parce que l'acte littéraire lui-même constitue une série de libertés prises vis-à-vis de l'hégémonie de la langue-système ${ }^{3}$. D'où la difficulté (ou les difficultés) qui pèse (pèsent) sur le traducteur du texte littéraire. Et Akakuru ${ }^{4}$ l'a bien remarqué lorsqu'il affirme, parlant de la traduction des textes littéraires que: «Traduire, pour ce qui est des textes littéraires, c'est revoir d'un œil second ce qui a été vu et formalisé par un œil et une culture premiers. C'est établir un réseau de sens à partir d'un réseau déjà existant...» Or, on sait que cet objet littéraire à traduire ne se présente pas de façon directe. Il adopte, pour naître, les formes de «masques» (Bestman 1980) ${ }^{5}$ : il est fond et forme, cette dernière étant du coup, plus que partout ailleurs, la marque distinctive de son être. Voilà que le style devient un élément conscient et entre irrémédiablement dans le processus de structuration, donc de signification littéraire. Certes, le style, c'est la forme dominante du texte, conditionnée par son inspiration et par sa destination, le tout subordonné à l'espace-temps de production ${ }^{6}$. Mais le style est

Meta, XLII, 4, 1997 
aussi la résultante de la personnalité de l'écrivain, le signe d'une humeur, une série d'obsessions individuelles 7 qui se manifestent au niveau du texte par des choix ponctuels d'éléments linguistiques, par des idiosyncrasies de structures et de diction. Domaine de prédilection de la stylistique qui «étudie les faits d'expression ${ }^{8}$ »comme conséquence et comme cause.

Ainsi, si la traduction littéraire vise le fond et les formes, la stylistique - étude des formes - devient alors une partie intégrante de cette tentative astreignante de restituer dans une langue seconde des formes qui impliquent, dans le réseau du système linguistique de départ, des effets et des sens. Et c'est là que se situe la justification de cette communication : analyser la traduction d'Arrow of God - roman d'Achebe - pour déterminer dans quelle mesure le processus de traduction a influencé (ou modifié tant soit peu) le style, voire l'orientation de l'œuvre originale.

Le roman qui fait l'objet de notre étude est le troisième de l'écrivain nigérian Chinua Achebe; il fut traduit en français aux éditions Présence Africaine en 1978 sous le titre La flèche de Dieu. On a remarqué déjà que la langue anglaise du roman original est calquée sur la façon de parler typiquement igbo. Ainsi, on affirme que les structures syntaxiques jusqu'à la vie des personnages s'inspirent de cette vision du monde igbo ${ }^{9}$.

C'est dire que le roman vise un public igbophone, du moins des lecteurs qui connaissent plus ou moins la manière d'être et de signifier de ce monde; même si la langue d'expression privilégiée est l'anglais. Et l'on voit que traduire en français, c'est chercher à recréer la tension «igbo/anglais» en une tension «igbo/français», et ce, à partir de l'anglais de l'original. Or, ce qui complique la situation du traducteur, c'est le fait que l'on est dans le domaine culturel, domaine où se pose avec une acuité réelle le problème de l'individualité de chaque culture et donc de ses modes d'expression. Car, si l'on peut trouver l'équivalent adéquat pour des textes pragmatiques (techniques, mathématiques, médecine, etc.), la réalité est autre pour des textes littéraires où une partie importante du message passe par les sonorités, le rythme, les intonations et les connotations.

Cette nature particulière du texte littéraire - catégorie à laquelle appartient notre texte de travail - impose au traducteur les impératifs de la littéralité ${ }^{10}$. Il ne s'agira plus de faire un choix entre une traduction pragmatique, visant tout simplement le message et laissant en marge le contenu culturel et la forme grammaticale; il ne s'agira pas non plus de faire une traduction esthético-poétique, qui trahira l'esthétique du modèle au profit de l'esthétique du jour. Ni de privilégier une traduction linguistique au détriment des considérations ethnographiques ${ }^{11}$. Le contexte culturel, message, les dominants formels et les connotations sont tour à tour indispensables si le praticien doit réussir son entreprise.

Ainsi le discours littéraire est-il d'emblée marqué par une complexité congénitale. Car cette langue d'Achebe - un anglais irrigué d'igbo - ne se réalise qu'au sein d'une culture, d'une expérience. Ce qui pousse à constater que le traducteur qui ose affronter l'anglais d'Achebe le fera en sachant que la fidélité, en ces matières, fait problème. Et l'on se demande, pour parler comme le poète martiniquais Léon Laleau, dans son poème Trahison, si l'on peut changer de langue sans changer d'effets: si l'on peut modifier les effets sans trahir ${ }^{12}$.

Dans la deuxième partie, nous avons pris au hasard la traduction des séquences qui frappent par le problème particulier des «formes travaillées» qu'elles posent: métaphores, tournures idiomatiques, mots isolés reliés à la culture génératrice de l'œuvre, etc. Et cela, dans le but de les analyser en vue de tirer des conclusions qui répercutent sur les problèmes généraux de la traduction et les effets de la traduction sur l'intégrité stylistique et sémantique de l'œuvre originale. 
Commençons notre discussion par la traduction des métaphores dans le roman d'Achebe. Pour la commodité de notre exposé, nous disposerons en forme de tableau bipartite les textes en anglais et en français.

La métaphore (comparaison condensée) :

On vient d'annoncer l'apparition de la nouvelle lune; les enfants comme les adultes, chez Ezeulu, vont la saluer. Les exemples sont tirés de cette scène.

\begin{tabular}{|c|c|}
\hline Extrait 1 & \\
\hline $\begin{array}{l}\text { Texte anglais } \\
\text { - I did not ask you, ant-hill nose. } \\
\text { - You will soon cry, long throat. }\end{array}$ & $\begin{array}{l}\text { Texte français } \\
\text { - Je ne t'ai rien demandé, toi dont le nez est } \\
\text { long comme une fourmilière. } \\
\text { - Tu vas pleurer, cou démesuré. }\end{array}$ \\
\hline $\begin{array}{l}\text { Analyse des données (extrait 1) } \\
\text { ant-hill nose } \\
\text { - Expression métaphorique: comparaison qui } \\
\text { met en jeu la connaissance de la taille de la } \\
\text { fourmilière. La perspective sémantique porte } \\
\text { ici sur la grosseur. Le processus de signifi- } \\
\text { cation sollicite la participation active du lecteur. }\end{array}$ & $\begin{array}{l}\text { «toi dont le nez est long comme une } \\
\text { fourmilière» } \\
\text { - Comparaison explicite qui fait perdre la } \\
\text { force et l'acuité de la métaphore de l'original. } \\
\text { Ce qui est mis en jeu ici est la «longueur», } \\
\text { non la «grosseur». cette formule est moins } \\
\text { littéraire que celle de l'original. La partici- } \\
\text { pation du lecteur / traducteur est limitée à la } \\
\text { simple recherche des significations. Perte de } \\
\text { l'image-choc. }\end{array}$ \\
\hline $\begin{array}{l}\text { long throat } \\
\text { - Expression métaphorique qui veut dire } \\
\text { «gourmand» et qui ne devait pas être prise } \\
\text { au pied de la lettre. Le processus de signifi- } \\
\text { cation est indirect et littéraire. }\end{array}$ & $\begin{array}{l}\text { «cou démesuré» } \\
\text { - Expression littérale. Constitue un contre- } \\
\text { sens saillant par rapport au sens de l'original. } \\
\text { Le statut de figure de l'original est détruit. }\end{array}$ \\
\hline
\end{tabular}

On constate ainsi certaines dérivations par référence au texte original en anglais. Voyons un autre extrait où l'on relève tour à tour des modifications sur des mots isolés et des constructions grammaticales ou rythmiques:

\section{Extrait 2}

Texte anglais

a) Ezeulu rose from his goatskin...

b) His ikenga, about as tall as a man's forearm. Its animal horn as long as the rest of its human body...

c) They told him to call it Name-sake and he did.

d) Ezeulu thought for a while as though he was unprepared for the proposal. Then he asked Obika how much on his new homestead was still undone.

e) Only the woman's barn, he replied ...There will be no cocoyam to put into it until harvest time.
Texte français

a) Ezeulu se leva de la peau de mouton...

b) Son ikenga (presqu'aussi long que l'avantbras d'un homme) dont la corne animale aussi longue que le reste de son corps humain...

c) On lui avait demandé de l'appeler «Homonyme» c'est ce qu' il fit.

d) Ezeulu réfléchit un instant comme s'il ne s'attendait pas à cette suggestion. Puis il demanda à Obika quels travaux il restait à faire dans sa nouvelle ferme.

e) Il ne reste que le grenier de la femme, répondit-il... On ne pourra pas y planter de taros avant la récolte. 
f) Nothing will wait, said Ezeulu. A new wife should not come into an unfinished homestead.. f) Rien n'attendra, dit Ezeulu. Une nouvelle femme ne doit pas entrer dans une ferme...

\section{Analyse des données (extrait 2)}

Dans notre relevé (a), goatskin est rendu par peau de mouton. Or, chez les Igbo, c'est plus souvent les chèvres (ou les poulets) qui sont exigés pour la plupart des rites et des cérémonies propitiatoires. Pourtant, nous savons que dans la perspective judéochrétienne, le mouton est valorisé. Ce qui explique (sans pour autant justifier) le choix de mouton pour dire chèvre. Autrement dit, les traductrices ont procédé à une adaptation, de manière à insérer le roman d'Achebe dans la vision du monde de la langue-cible.

Dans la traduction du segment (b), l'emploi de long pour le tall de l'original et l'introduction des parenthèses invitent nos remarques. D'abord, nous ne voyons pas de problème à ce que la traduction se réalise sans parenthèses en respectant la forme de l'original à peu de modifications près. D'ailleurs, la présence des parenthèses semble donner l'impression, fausse, que l'information contenue dans la séquence est secondaire. En ce qui concerne le mot long qui remplace tall, rien n'égale cette infidélité sémantique. L'ikenga se voit attribué tall parce qu'on lui accorde le statut d'homme: c'est la hauteur, et non la longueur qui est ici visée.

Notre relevé (c) présente un autre problème. On se demande la justification de l'emploi du présentatif $c^{\prime}$ est dont la forme appartient plus au discours qu'à l'énonciation historique. Pourquoi pas ce qu'il fit, tout simplement, forme qui maintient, de plus, le rythme de l'original.

On trouve ainsi que ces libertés prises vis-à-vis de l'original empirent surtout dans les cas des exemples (d), (e), (f) où des mots isolés mal traduits entraînent une désorientation des énoncés concernés. Pour la commodité de notre exposé, disposons les données sous forme de tableau:

Analyse des données (d), (e), (f)

Dans (d) proposal dont le verbe en français est proposer qui veut dire, selon le Littré: mettre une chose en avant pour qu'on en délibère la valeur, signifie qu'Edogo soumet un projet quelconque à son père. Projet sur lequel ce dernier doit donner son avis.

Pour ce qui est de homestead, il s'agit, selon le contexte du roman, d'une case, une concession bâtie par un homme pour sa famille.

Dans (e), barn est un endroit où l'on met les produits de la terre. On n'y plante rien. Et quant à l'expression idiomatique put into, elle équivaut à déposer, garder, stocker, etc., car le barn est un dépotoir.
Le mot suggestion est choisi au licu de proposition. Or, le verbe suggérer a un sens souvent négatif. La traduction aurait été plus heureuse si le mot proposition avait été employé.

Le choix de ferme pour traduire homestead est un cas de sous-traduction qui entraîne un contre-sens par rapport à l'idée de l'original. Le mot case convient plus que ferme. Une ferme étant un lopin de terre cultivée ou cultivable, non une forme de maison à habiter.

Le mot grenier fait problème. Le contexte linguistique dans lequel figure ce mot (dans la version française) donne l'impression qu'on peut planter dans le grenier. Cette déformation de sens vient de ce que l'expression idiomatique put into (garder, déposer...) est traduit maladroitement par planter. 
Viennent enfin, dans (f), should not et come into. Should not est soigné et n'est pas du tout agressif. Come into sous-tend l'idée de s'installer, ou même d'habiter.
Ne doit pas est un peu trop sec et, partant, plus agressif que l'original. Ne devrait pas conviendrait mieux. Entrer perd toute cette finesse et détruit le statut de plénitude de l'expression originale.

Si nous nous reportons au contexte sous-jacent (linguistique et extralinguistique) de l'emploi des mots/expressions relevés, nous nous rendons compte du coup que grenier, should be et come into accusent une modification de sens que ne justifie aucune logique du texte original.

De même, dans les cas de put into et ensuite de should not, les perspectives du texte anglais sont viciées. En pays igbo, on ne plante rien dans les greniers. Et cette réalité aurait guidé le traducteur dans la reformulation de put into. C'est en réalité la non-maîtrise de certains éléments de la culture des locuteurs (voire du texte dans sa totalité) qui entraîne ce genre de traduction linguistique qui ignore de façon évidente la souplesse qu'une familiarité avec la culture igbo devrait faciliter dans le rendement de should not par ne doit pas.

Extrait 3: Expressions idiomatiques / formes et valeurs temporelles. Une expression idiomatique mal traduite ou même une valeur temporelle mal saisie et, partant, mal rendue (par rapport à la valeur stylistique du texte original), peut conduire au contresens ou modifier les effets envisagés de l'énoncé. Nous tirons nos exemples de la scène, dans le roman d'Achebe, où le capitaine Winterbottom donne une recommandation à son adjoint qui part en tournée dans la région:

\begin{tabular}{|c|c|}
\hline $\begin{array}{l}\text { Texte anglais } \\
\text { i) ...I wouldn't put anything past Wright } \\
\text { from sleeping with native women... } \\
\text { ii) When you are in Umuaro... } \\
\text { iii) ...ugly stories of whipping }\end{array}$ & $\begin{array}{l}\text { Texte français } \\
\text {...je n'accepterai rien de Wright en dehors du } \\
\text { fait qu'il couche avec les femme indigènes... } \\
\text { Lorsque vous serez à Umuaro... } \\
\text { Toutes sortes d'histoires horribles de séances } \\
\text { de fouet... }\end{array}$ \\
\hline $\begin{array}{l}\text { Analyse des données (extrait } 3 \text { ) } \\
\text { «...put anything past Wright» } \\
\text { Expression idiomatique qui signifie que } \\
\text { Monsieur Wright est capable de tout ce qui } \\
\text { est négatif. } \\
\text { «When you are in Umuaro» } \\
\text { Le présent are a la valeur du futur. Ceci relève } \\
\text { du style parlé. } \\
\text { «ugly stories of whipping» } \\
\text { Emploi direct en un mot isolé. }\end{array}$ & $\begin{array}{l}\text {...n'accepterai rien de Wright } \\
\text { expression littérale qui constitue un contre- } \\
\text { sens colossal et escamote la connotation } \\
\text { négative. } \\
\text { «Lorsque vous serez à Umuaro» } \\
\text { Cet énoncé explicite la valeur futur que l'ori- } \\
\text { ginal laisse implicite. } \\
\text { «...des séances de fouet» } \\
\text { Emploi de mots supplémentaires non motivés } \\
\text { par l'original. Un cas d'étoffement superflu. } \\
\text { Le mot bastonnade aurait suffi à restituer } \\
\text { whipping. }\end{array}$ \\
\hline
\end{tabular}

D'autres exemples de modifications des mots isolés, des expressions idiomatiques, des valeurs temporelles des verbes, de la modification du rythme, donc du sens à partir d'une erreur de ponctuation, etc., abondent dans la traduction française d'Arrow of God. Voyons rapidement un ou deux exemples de la valeur temporelle suivie par une modification au niveau de la ponctuation: 


\begin{tabular}{|l|l|}
\hline $\begin{array}{l}\text { iv) «I have already said that what this new } \\
\text { religion will bring wears a hat on the head.» }\end{array}$ & $\begin{array}{l}\text { J'avais déjà dit que ce que la nouvelle } \\
\text { religion amènerait... porterait un chapeau sur } \\
\text { la tête. }\end{array}$ \\
\hline $\begin{array}{l}\text { Analyse des données } \\
\text { I Have said: passé composé (temps du }\end{array}$ & $\begin{array}{l}\text { J'avais...dit: plus-que-parfait (temps du récit, } \\
\text { style pédant) } \\
\text { amill bring : futur simple (temps du discours) } \\
\text { wears: conditionnel présent de l'indicatif (implique la } \\
\text { certitude). }\end{array}$
\end{tabular}

La commutation du présent pour le futur (en igbo comme dans l'anglais de l'original) relève de l'oralité et n'implique d'aucune manière une prétendue absence en igbo (voire dans les langues africaines) de la concordance. Très souvent, la réalité future dont il est question, dans ces énoncés, est si patente et presque si palpable qu'elle fait déjà partie du présent réel du locuteur. Or, tout cela est détruit dans la version française. Et cela est peut-être imputable à ce que Walter Benjamin qualifie de pur langage et qui résulte d'une incapacité, chez le traducteur, à poser les rapports - non pas entre deux langues - mais entre deux discours ${ }^{13}$. D'où les modifications non voulues au niveau du sens et qui résultent de certaines fautes de ponctuation.

Extrait 4: Modifications au niveau de signes de ponctuation/rythme. Une virgule ou un point mal placé peut changer le sens d'un énoncé tout en modifiant le débit original de celui-ci. C'est le cas de la traduction de l'extrait suivant:

\begin{tabular}{|l|l|}
\hline $\begin{array}{l}\text { Texte anglais } \\
\text { - When an adult is in the house, the she-goat } \\
\text { is not left to suffer the pains of parturition on } \\
\text { its tether... }\end{array}$ & $\begin{array}{l}\text { Texte français } \\
\text { Lorsqu'un adulte est dans la case d'une } \\
\text { brebis, celle-ci ne souffre pas les douleurs de } \\
\text { l'enfantement abandonné à son piquet... }\end{array}$ \\
\hline $\begin{array}{l}\text { Analyse des données (extrait 4) } \\
\text { - La virgule est juste après house qui est } \\
\text { attribué à an adult. }\end{array}$ & $\begin{array}{l}\text { La virgule est après la case d'une brebis qui } \\
\text { fait croire que l'adulte est dans la case de } \\
\text { l'animal. Or, cette idée ne correspond pas à } \\
\text { celle de l'original. }\end{array}$ \\
\hline
\end{tabular}

Ainsi, un simple fait de ponctuation (de signe de ponctuation mal placé) pourrait aboutir à des contresens de la pire espèce, comme le montre l'exemple ci-dessus.

On remarque, ainsi, tout au long de la traduction du texte d'Achebe, des modifications, des modulations et des imprécisions par rapport au sens de l'original. Et puisque ces réalités entraînent des modifications de sens, on a le droit d'attribuer cet état de choses au problème de compréhension (pour parler dans l'optique du modèle séquentiel de Gile ${ }^{14}$. Or, dans certains cas, l'erreur vient de beaucoup plus loin. Elle vient, d'abord, d'une connaissance approximative de la culture igbo et de ses modes d'expression culturelle; elle vient ensuite - et cela est souvent minimisé - de l'absence d'une compétence poétique qui dénie au traducteur la sensibilité nécessaire pour capter l'étymon spirituel du texte source et pour enfin restituer dans le texte cible les éléments stylistiques de l'original.

\section{CONCLUSION}

Si la stylistique s'intéresse aux éléments du style d'un texte donné et si le style d'un texte implique une certaine stratégie de l'auteur à l'intention du destinataire, il s'ensuit 
que tout changement de la perspective discursive, toute modification de l'énoncé premier se répercutent sur le plan de la réception. Car dans la littérature plus que partout ailleurs, la valeur d'un énoncé va au-delà du linguistique et intègre les conventions littéraires (scripturales, idéologiques, etc.), qui surdéterminent le décodage de l'énoncé en question.

$\mathrm{Au}$ cours de ce travail, nous avons relevé des cas de modifications de la langue source, lesquelles entraînent, du même coup, des modifications au niveau du sens et du style. Nous avons, à ce titre, attiré l'attention sur la dé-métaphorisation (long throat/cou démesuré), sur des expressions idiomatiques mal perçues (put into/ «planter» au lieu de «garder»), sur des mots isolés mal traduits, des signes de ponctuation mal placés, des relatifs flottants, des nuances expressives perdues ou viciées (should not/ne doit pas; come into/entrer), etc. Ces modifications, qui sont des transgressions par rapport à l'original, concernent le plus souvent des mots / expressions qui sont contexte-dépendants ${ }^{15}$. Et cela se remarque, surtout, lorsque l'on doit opérer un choix entre la réalité culturelle (le mode d'expression) du lecteur du texte traduit et la fidélité au texte original. C'est ainsi que - pour s'aligner sur ce lecteur second - on a traduit peau de chèvre comme peau de mouton.

De plus, nous savons qu'en littérature, on ne peut réduire le sens d'un énoncé au lexical. La notion du symbolisme littéraire, la prédominance du rythmique et des formes indirectes, aboutit à privilégier ce que Saussure ${ }^{16}$ nomme valeur (mais ici allant au-delà de la langue pour atteindre le texte voire le discours) plutôt que le sens en tant que tel.

C'est dire que même si - dans l'ensemble - la traduction d'Arrow of God respecte l'esthétique de l'original, on remarque néanmoins un décentrement (discursif, voire rhétorique) qui résulte des substitutions et/ou des modulations dans les formes de l'œuvre originale. Or, les éléments que l'on violente, dans la pratique de la traduction, sont ceux qui participent au style de l'auteur, à ses stratégies particulières, à ses obsessions. Et c'est en les modifiant, en cherchant à leur imposer une nouvelle individualité, que le traducteur porte atteinte à l'intégrité de l'original. Voilà qu'en modifiant tant soit peu Arrow of God, nos traductrices ont accouché d'une flèche de Dieu qui n'est pas tout à fait La Flèche de Dieu d'Achebe.

\section{Notes}

1. McLuhan (1964).

2. Darbelnet (1977:7).

3. La littérature se situe sur le plan du discours, non de la langue-système. Elle implique très souvent ce que l'on qualifie de licence poétique.

4. Akakuru (à paraître).

5. Selon Bestman (1980) : «L'écriture littéraire apparaît comme un spectacle mythique codé, un jeu de masques ésotériques aux non-initiés [...] En définitive, l'art du roman est essentiellement un art dissimulateur, un jeu d'artifices, de déguisement, d'illusions ; écrivains et lecteurs sont rituellement complices...»

6. Nous pourrions dire que dans la constitution d'un style, l'auteur, le but du discours, les contraintes de la langue, l'espace / temps de production et le lecteur hypothétique sont des éléments déterminants. Cette définition dépasse le cadre étroit du style «c'est l'homme lui-même» pour réintégrer la perspective qui va de l'expressivité à l'impressivité.

7. Voir Barthes (1953).

8. Bally (1957: 16).

9. Killam (1977: 13).

10. La littérature est un message d'un type particulier. C'est un mode de communication qui déploie toute une batterie de stratégies subjectives et indirectes pour atteindre le lecteur. De ce fait, pouvoir «lire» littérairement un texte littéraire en vue de le traduire devient cuvre de spécialiste. Puisqu'il faudra se pourvoir de ce que l'on désigne comme compétence poétique : la capacité à saisir le discours littéraire (son message, son expressivité et impressivité, ses procédures dissimulatrices). Le littéraire, c'est le langage qui se met en scène. La littérarité, c'est cette qualité d'être littéraire.

11. Vu que le fait littéraire naît au carrefour du fait et de l'émotion, du message et des formes, toute traduction du littéraire doit procéder de sorte à restituer, au niveau du texte traduit, toutes les ressources intellectuelles et esthétiques qui définissent l'objet littéraire. 
12. Laleau (à parâtre). Nous avons opéré tout simplement un calque syntaxique sur la formule véritable. Voir Kesteloot (1963).

13. Walter Benjamin (1971) critique une tendance qu'il qualifie de pur langage et qui consiste à infléchir le texte original dans le sens de la langue d'arrivée au lieu de garder l'esprit de l'original au cours de la traduction. Écoutons-le: «Nos versions, même les meilleures [...] veulent germaniser le sanscrit, le grec, l'anglais, au lieu de sanscritiser, d'helléniser, d'angliciser l'allemand...»

14. Voir Gile (1992), notamment pp. 253 et 255 . Nous faisons référence, notamment, au modèle séquentiel que l'auteur nous propose comme outil pragmatique d'enseignement et donc d'évaluation de l'apprentissage.

15. Il s'agit ici des éléments linguistiques / non-linguistiques qui relèvent spécifiquement de la culture igbo et peuvent, de ce fait, poser le problème d'intraduisibilité - concept qui soulève actuellement beaucoup de controverse. Voir à ce titre Newmark (1982).

16. La notion de valeur, telle que Saussure (1916) nous la propose, réside en langue. Elle ne prend pas en compte, par conséquent, le poids du social et de l'historique dans le processus de signification. C'est en réintégrant la valeur dans le discours que nous pouvons arriver à la véritable portée sémantique d'un texte littéraire donné.

\section{RÉFÉRENCES}

AKAKURU, I. A. (à paraître) : «Traduire et signifier : la problématique du transcodage».

BALLY, Charles (1957): Traité de stylistique française, $3^{e}$ édition, Paris, Klinksieck.

BARTHES, Roland (1953): Le degré zéro de l'écriture, Paris, Seuil.

BENJAMIN, Walter (1971): CEuvres Í, Mythe et violence, Paris, Denoël, p. 274.

BESTMAN, Martin T. (1980): Le jeu des masques, Québec, Éditions Nouvelles Optiques, cité par Nwanne Mkpa, Traduction de quatre chapitres de "Le jeu des masques» de Martin Bestman, Université de Port Harcourt, TER de maîtrise, $2^{\mathrm{e}}$ partie, 1990, p. 2.

DARBELNET, Jean (1977) : «Niveaux de la traduction», Babel, XXIII-1.

GILE, Daniel (1992) : «Les fautes de traduction: une analyse pédagogique», Meta, 37-2, pp. 251-262.

KESTELOOT, Lilyan (1963): Les écrivains noirs de la langue française : naissance d' une littérature, Bruxelles, Édition de l'Université de Bruxelles.

KILLAM, G. D. (1977): The Writing of Chinua Achebe, Revised Edition, Ibadan, Heinemann.

LALEAU, Léon (à paraître) : Trahison, cité par I. A. Akakuru, «Traduire et signifier : la problématique du transcodage».

MCLUHAN, Marshall (1964): Understanding the Media (The Extensions of Man), New York, Signet Books.

MESCHONNIC, Henri (1981): «Traduire la Bible, de Jonas à Jona», Langue française, $\mathrm{n}^{\circ} 51$, septembre, Paris, Larousse, pp. 35-52.

NEWMARK, Peter (1982) : Approaches to Translation, Oxford, Pergamon Press, p. 9.

SAUSSURE, Ferdinand de (1916) : Cours de linguistique générale, Lausanne, Payot, 331 p., nouvelle édition, 1972, $532 \mathrm{p}$. 